\title{
The Difference of Endometrial Vascular Endothelial Growth Factor (VEGF) Expression between Polycystic Ovarian Syndrome (PCOS) and Normal Women
}

\author{
Ricky Bernadi Saputra, Uki Retno Budihastuti, Sri Sulistyowati, \\ Eriana Melinawati, Supriyadi Hari Respati
}

Department of Obstetrics and Gynecology, Faculty of Medicine, Universitas Sebelas Maret/ Dr. Moewardi Hospital, Surakarta

\section{ABSTRACT}

Background: Polycystic Ovarian Syndrome (PCOS) is an endocrine disorder in women characterized by increased levels of androgens, ovulatory dysfunction, and Polycystic Ovarian Morphology (PCOM), as well as a combination of classic clinical features. Women with PCOS experience changes in the endometrium and endometrial receptivity markers such as VEGF (Vascular Endothelial Growth Factor). VEGF increases the permeability and dilation of endometrial blood vessels which play an important role in implantation. This study aimed to analyze the difference of VEGF expression between women with PCOS and normal women.

Subjects and Method: This study was a casecontrol study design conducted at the polyclinic of the Department of Obstetrics and Gynecology, Dr. Moewardi Hospital, Surakarta, Central Java, in July 2020. There were 30 infertile women with PCOS (diagnosis based on Rotterdam criteria, consensus 2003) and 30 women without PCOS as the sample of this study. The dependent variable was VEGF expression. The independent variables were age, BMI, family history with PCOS, menarche, occupation, and education. VEGF expression was obtained from an endometrial biopsy on days
19-24 of periods. The differences in VEGF expression (in \%) were analyzed by the Mann-Whitney test. VEGF determinants were analyzed by a multiple linear regression.

Results: The mean of VEGF expression in women with PCOS (Mean=64.33; $\mathrm{SD}=44.43)$ was higher than in women without PCOS (Mean= 7.90; $\mathrm{SD}=8.73$ ) and the result was statistically significant $(\mathrm{p}<0.001)$. $\mathrm{PCOS}(\mathrm{b}=42.09 ; 95 \% \mathrm{CI}=-$ 64.26 to $-19.93 ; \mathrm{p}<0.001)$ and obese $(\mathrm{b}=38.63$; $95 \% \mathrm{CI}=-63.44$ to $-13.82 ; \mathrm{p}=0.003$ ) increased VEGF expression.

Conclusion: VEGF expression in the endometrium of women with PCOS is higher than normal women.

Keywords: VEGF, endometrium, Polycystic Ovarian Syndrome

\section{Correspondence:}

Ricky Bernadi Saputra. Department of Obstetrics and Gynecology, Faculty of Medicine, Universitas Sebelas Maret/ Dr. Moewardi Hospital, Surakarta, Indonesia. Telp: o81221161933 Email: rickysaputradr@gmail.com

\section{Cite this as:}

Saputra RB, Budihastuti UR, Sulistyowati S, Melinawati E, Respati SH (2020). The Difference of Vascular Endothelial Growth Factor Expression between Women with Policystic Ovarium Syndrome and Normal Women. Indones J Med. 05(03): 230-239. https://doi.org/10.26911/theijmed.2020.05.03.08.

(i) (2) Indonesian Journal of Medicine is licensed under a Creative Commons

EY NC SA Attribution-Non Commercial-Share Alike 4.o International License.

\section{BACKGROUND}

Polycystic Ovarian Syndrome (PCOS) is an endocrine disorder in women characterized by increased levels of androgens, ovulatory dysfunction, and Polycystic Ovarian Morphology (PCOM), as well as a combination of classic clinical features including obesity, hirsutism, alopecia, acne, irregular periods, infertility, and high blood pressure (Schneider et al., 2019). PCOS was found in $5 \%$ to $50 \%$ of women of reproductive age, which affected 5 million women in the United States. In Indo- 
nesia, the highest frequency of women with PCOS was women aged 26-30 years old, which was $45.7 \%$ (Sumapraja et al., 2011). PCOS most often manifested as oligomenorrhea or amenorrhea due to ovulatory dysfunction in $70 \%$ of women, accompanied by symptoms of androgen excess, such as acne and hirsutism. Classic Polycystic Ovarian Morphology (PCOM) was found at varying levels. It could occur in women without other classic metabolic symptoms. PCOS could be diagnosed since puberty or found during infertility or anovulation examination (Ring, 2017).

Generally, in most cases, infertility related to PCOS was caused by anovulation. Besides, endometrial dysfunction also affected subfertility in patients with PCOS, characterized by changes in endometrial histomorphology and endometrial receptivity markers, including: 1) estrogen, progesterone, and various receptors in it; 2) growth factor (GF), Insulin-like Growth Factor-1 (IGF-1), Vascular Endothelial Growth Factor (VEGF); 3) cytokines such as Leukemia Inhibitory Factor (LIF); Matrix Metalloproteinase-9 (MMP-9); Interleukin-11; Colony Stimulating Factor-1 (CFS-1); Cyclooxygenase-2 (COX-2); 4) modulator for cell attachment: MUC-1, Integrin, Basigin (BSG); and 5) developmental factors (Homeobox genes) (Yoshii et al., 2013).

Vascular Endothelial Growth Factor (VEGF) is a homodimeric glycoprotein that binds to high-affinity receptors on endothelial cells, mediates angiogenesis and vascular permeability, and is secreted by granulosa lutein cells of ovary and theca cells, and endometrium. VEGF plays an important role in ovarian folliculogenesis and normal reproductive function. Women with PCOS experienced an increase in serum VEGF levels, as well as an increase in the flow rate in ovarian vessels detected by Doppler. High vascularization could cause abnormal growths from the theca interna, where there was androgen steroidogenesis, thus causing hyperandrogenism, a characteristic of PCOS (Almawi et al., 2016). Many growth factors are involved in angiogenesis, but VEGF is considered the most important because it increases the permeability and dilation of endometrial blood vessels which play an important role in implantation. This study was conducted to determine the VEGF expression in the endometrium of women with PCOS which was expected to be higher than normal women. The disruption of the implantation process occurred due to abnormal adhesion mechanisms.

\section{SUBJECTS AND METHOD}

\section{Study Design}

This study was a case-control study design conducted at Dr. Moewardi Hospital, Surakarta. The study subjects were selected from July 2020 until the number of subjects was met. This study used the Anatomy Pathology Laboratory of Dr. Sardjito Hospital to examine VEGF expression using Immunohistochemistry (IHC) test.

\section{Population dan Sample}

The study subjects were the sufferers of PCOS, the infertile women who went to the polyclinic of Dr. Moewardi Hospital, Surakarta, from July 2020 until the number of subjects was met.

The control group consisted of women who underwent laparoscopy or laparotomy and were not diagnosed with PCOS, underwent Female Sterilization, underwent regular IVA test check-up, had undergone anamnesis and gynecological examination, and women who were not suspected of having PCOS (fertile, no history of pelvic pain, no dysmenorrhea, no dyspareunia, and normal result of gynecological clinical examination). The study subjects were selected according to the inclusion criteria. The study subjects were excluded based on the exclusion criteria. 
The inclusion criteria were infertile patients with PCOS who came to Dr. Moewardi Hospital days 19-24 of periods (28-day cycle), 12 days from the next cycle if the menstrual cycle was short or 10 days from the next cycle if the menstrual cycle was long; aged 21-40 years; and patients who did not have contraindications to surgery.

The exclusion criteria were patients who used hormonal birth control (KB); had a malignant disease; had received medical treatment for PCOS in the past 6 months; get Hormone Replacement Therapy (HRT) for the past 6 months; consumed Non-Steroidal Anti-Inflammatory Drugs (NSAIDs) in the past month.

\section{Study Variables}

The dependent variable was the VEGF expression in the endometrium. The independent variable was the diagnosis of PCOS. The external variables were family history with PCOS, age at menarche, Body Mass Index (BMI), age, type of occupation, and level of education.

\section{Operational Definition of Variables} The diagnosis of PCOS was established by the Rotterdam 2003 criteria if there were two of three symptoms, by looking at the patient's medical record data determined by a specialist.

VEGF expression in the endometrium was luminal epithelial cells and glandular that described VEGF on Immunohistochemistry (IHC) test. The positive color result of VEGF was brown color, while for the negative color result was pale color counted. The results were compared per 200 luminal epithelial cells and glandular in one field of view. It was assessed in a percentage. Family history with PCOS was a hereditary factor of parents who could be one of the risk factors that could not be modified in the emergence of several diseases such as the PCOS disease that was found in family members of patients with PCOS.
Age at menarche was the first time women got periods.

BMI was used to determine the amount of fat stored in the body by comparing body weight (in kilograms) divided by the square of height (in meters) where the increase in BMI was associated with risk factors for diabetes mellitus and cardiovascular disease. This study used the calculation of BMI according to WHO 2004 for Asian population that was in accordance with the study subjects. The study subjects were classified into 2 groups: obese and non-obese group (Sachdeva et al., 2019).

Age was classified based on the opinion that the majority of women with PCOS who experienced infertility aged $21-40$ years. The ideal age to get married and have children for women was 21 years.

Type of occupation was classified into two groups: those who had formal jobs and those who formally did not have jobs.

The education level was classified based on the last level of the education they had, consisting of basic education (elementary school/junior high school) and further education (senior high school / college level).

\section{Data Analysis}

This study used a chi-square test for bivariate analysis. This study used a linear regression model for multivariate analysis. Its significance was considered with a value of $\mathrm{p}<0.05$

\section{Research Ethics}

This study was conducted based on the study ethics which consisted of an informed consent form, anonymity, confidentiality. The ethical clearance in this study came from the Health Research Ethics Committee of Dr. Moewardi Hospital, Surakarta, Central Java, No: 842/VII/HREC/202O.

\section{RESULTS}

\section{Sample characteristics}

Table 1 showed sample characteristics. Table 1 showed that two third of samples were at 
Saputra et al. / The Difference of Vascular Endothelial Growth Factor Expression

age $<37$ years. As many as $10 \%$ women had

family with history of PCOS.

Table 1. Sample characteristics

\begin{tabular}{llcc}
\hline & Variable & Frequency & Percentage (\%) \\
\hline Age (years) & $<$ 37 years & 40 & 66.6 \\
& $\geq 37$ years & 20 & 33.3 \\
Occupation & Unemployed & 34 & 56.6 \\
\multirow{2}{*}{ Education } & Employed & 26 & 43.3 \\
\multirow{2}{*}{ Family history of PCOS } & 24 & 40.0 \\
\multirow{2}{*}{ Age at menarche } & < Senior High School & 36 & 60.0 \\
\multirow{2}{*}{ BMI $\left(\mathrm{kg} / \mathrm{m}^{2}\right)$} & No & 54 & 90.0 \\
& Yes & 6 & 10.0 \\
& $<14$ years old & 26 & 43.3 \\
& $\geq 14$ years old & 34 & 56.6 \\
& Normal & 44 & 73.3 \\
\hline
\end{tabular}

\section{Bivariate analysis}

Table 2 showed the difference of VEGF expression between women with PCOS and normal women. Table 2 showed that VEGF

expression (in \%) in women with PCOS (Mean= 64.33; $\mathrm{SD}=44.43)$ was higher than in normal women $(\mathrm{Mean}=7.90 ; \mathrm{SD}=8.73)$ with $\mathrm{p}<0.001$.

Table 2. The Difference of VEGF expression between PCOS and normal women

\begin{tabular}{lcccc}
\hline \multicolumn{1}{c}{ Group } & N & Mean & SD & p \\
\hline VEGF in women with PCOS (\%) & 30 & 64.33 & 44.43 & $<0.001$ \\
VEGF in normal women (\%) & 30 & 7.90 & 8.73 & \\
\hline
\end{tabular}

Table 3 showed the difference of the variables between women with PCOS and normal women. Table 3 showed that women with family history of PCOS had the likelihood to experience PCOS 2.15 times than those without family history of $\mathrm{PCOS}(\mathrm{OR}=2.15$; $\mathrm{p}=0.671)$. Obese women increased the risk of $\operatorname{PCOS}(\mathrm{OR}=6.88 ; \mathrm{p}=0.040)$.

Table 3. The difference of the variables between women with PCOS and normal women

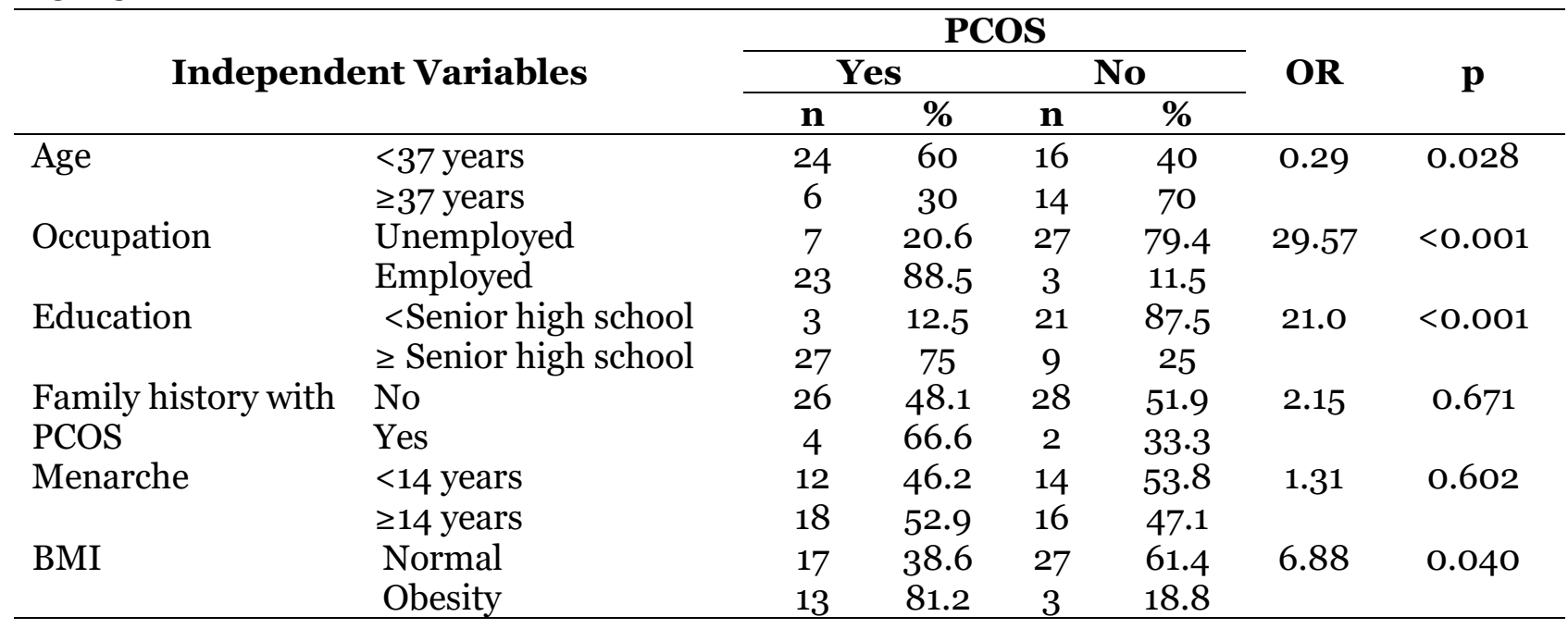

3. The results of multivariate analysis Table 4 showed the results of multivariate analysis on the determinants VEGF express- ion. Table 4 showed that $\mathrm{PCOS}(\mathrm{b}=0.50 ; 95 \%$ $\mathrm{CI}=-64.26$ to $-19.93 ; \mathrm{p}<0.001)$, had family history with $\mathrm{PCOS}(\mathrm{b}=0.22 ; 95 \% \mathrm{CI}=-62.74$ 
to -36.32; $\mathrm{p}=0.054)$, and obesity $(\mathrm{b}=0.40 ; \quad$ increased the risk of higher VEGF expression. $95 \% \mathrm{CI}=-63.44$ to $-13.82 ; \mathrm{p}=0.003)$

Table 4. Multivariate analysis on the determinants VEGF expression

\begin{tabular}{lcccc}
\hline \multirow{2}{*}{ Independent Variable } & Regression & \multicolumn{2}{c}{ 95\%CI } & \multirow{2}{*}{ p } \\
\cline { 3 - 4 } & coefficient (b) & Lower limit & Upper limit & \\
\hline PCOS & 0.50 & -64.26 & -19.93 & $<0.001$ \\
Age & 0.14 & -37.86 & -11.99 & 0.303 \\
Occupation & 0.31 & -1.17 & -54.62 & 0.060 \\
Education & 0.15 & -10.15 & -36.13 & 0.265 \\
Family history with PCOS & 0.22 & -62.74 & -36.32 & 0.054 \\
Menarche & 0.04 & -18.60 & -25.46 & 0.756 \\
BMI (obese) & 0.40 & -63.44 & -13.82 & 0.003 \\
\hline
\end{tabular}
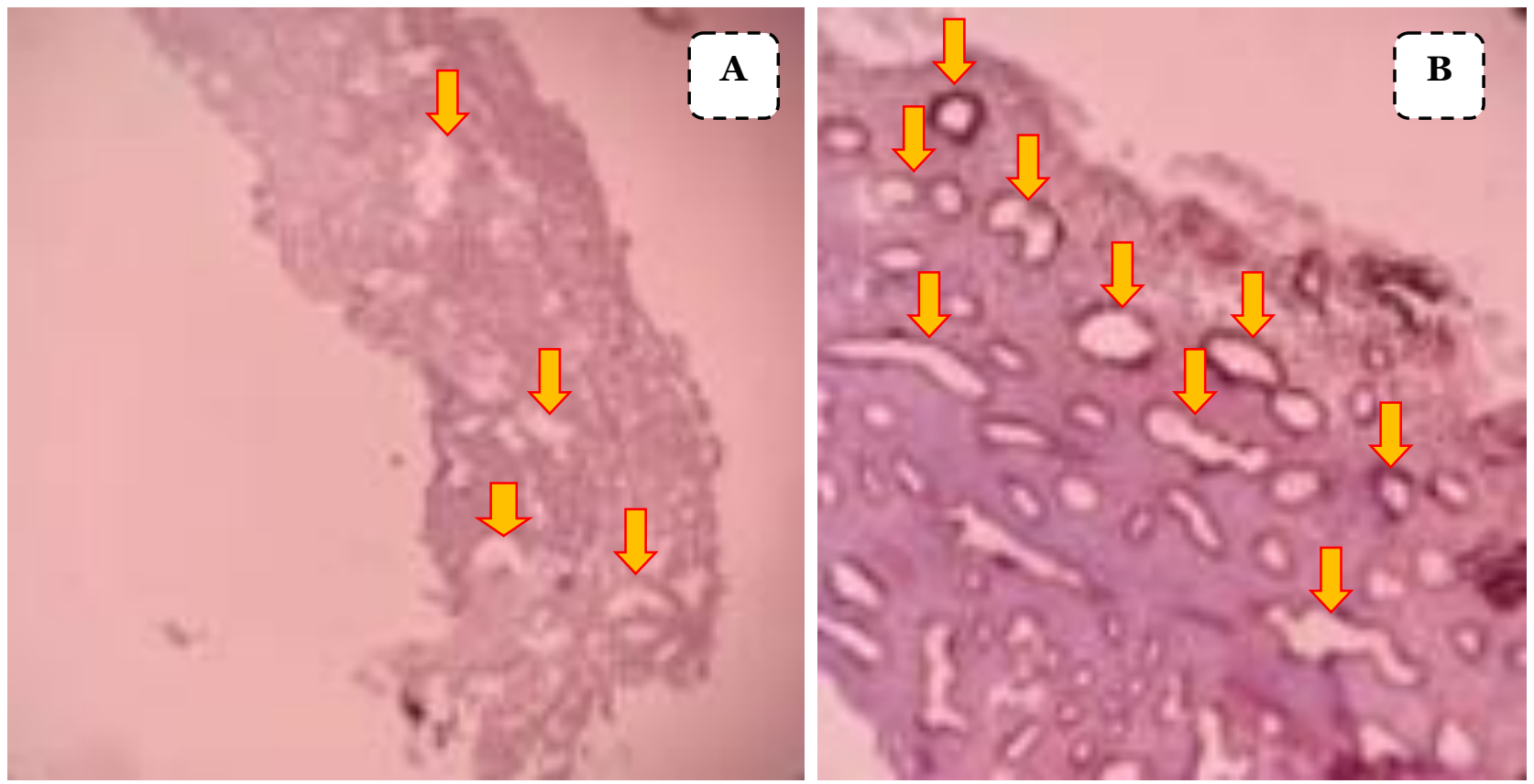

Figure 1. The immunohistochemistry (IHC) examination with 10 times magnification to examine endometrial VEGF expression.

\section{(A) Control Group and (B) PCOS Group}

Figure 1 depicted the immunohistochemistry (IHC) examination with 10 times magnification to examine endometrial VEGF expression between women with PCOS and normal women. The VEGF expression in the endometrium was in the form of luminal epithelial cells and glandular. It described VEGF on Immunohistochemistry (IHC) examination by doing 10 times magnification. The positive color result of VEGF was brown color while for the negative color result was pale color as shown in figure A.

\section{DISCUSSION}

PCOS is a common endocrinopathic condition found at reproductive age. It manifests clinically in a broad spectrum of disorders from mild ovulation disorders to typical syndromes characterized by obesity, hyperandrogenism, and insulin resistance. One of the fundamental features of PCOS is the loss of the mechanism of intra-ovarian autoregulation which causes unifollicular ovulation as observed in normal ovaries (Peitsidis and Agrawal, 2010). 
PCOS was one of the causes of infertility in women. It is estimated that around 5$10 \%$ of women of reproductive age suffered from PCOS, $75 \%$ of patients with anovulatory infertility. Patients with PCOS were mainly obese, had metabolic syndrome, had androgen excess, ovulatory dysfunction, infertility, etc. Endometrial receptivity was the receptive ability of the maternal endometrium to the blastocyst, which might be greatly affected in PCOS condition. Endometrium with good receptivity allowed the blastocyst to reach the location, adhesion, implantation, and other oxidation processes. Persistent low endometrial receptivity made it difficult to nastasi blastocysts in the endometrium, thus causing infertility in patients. The result showed that PCOS caused a low level of endometrial receptivity, seriously affected the oxidation and success level of embryo transfer (Zhao et al, 2019).

VEGF showed a close correlation with endometrial receptivity. It also participated in placental angiogenesis. Therefore, it was considered an important marker of endometrial receptivity. Several studies have shown that endometrial blood flow affected endometrial receptivity. As a result, VEGF was considered to correlate with endometrial receptivity. The level of VEGF expression could directly reflect the volume of uterine blood flow. Studies have shown that VEGF gave a specific effect on vascular endothelial cells of the endometrium, which was able to promote the proliferation and migration of vascular endothelial cells and increase vascular permeability. VEGF played an important role in endometrial neovascularization (Zhao et al, 2019).

The human endometrium had regular growth and damage cycles. It also had physiologically significant angiogenesis. Angiogenesis was the basis for the development and differentiation of endometrium for implanttation. These vascular changes were consi- dered to be mediated by VEGF and the specific receptors. Six members of the VEGF family, namely VEGF-A, VEGF-B, VEGF-C, VEGF-D, VEGF-E, and placental growth factor (PlGF) have been identified. VEGF was a heparin-binding glycoprotein and endothelial cell mitogen that caused increased vascular permeability. It was mediated after giving human chorionic gonadotrophin during the ovarian stimulation cycle. VEGF was described in granulosa lutein cells, ovarian theca cells, and uterine endometrium. VEGF also played an important role in cyclic angiogenesis and regulation of vascular permeability, which was very important for ovarian folliculogenesis. Serum VEGF concentration increased in the luteal phase of the period cycle and VEGF was suppressed by down-regulation of the reproductive axis (Lai et al., 2015).

VEGF played an important role in normal reproductive function. The abnormalities in ovarian angiogenesis could be seen in women with PCOS as well as in ovulation disorders, subfertility, and pathogenic conditions such as endometriosis. Increased VEGF expression in the dense hypoechoic stroma in polycystic ovaries associated with increased ovarian stroma blood flow could be the cause of loss of intra-ovarian autoregulation. This mechanism was shown in women with PCOS.

Women with PCOS experienced an increase in VEGF expression, as well as an increase in the flow rate in the ovarian vessels detected by Doppler. High vascularrization could cause abnormal growths of the theca interna, where there was androgen steroidogenesis, thus causing hyperandrogenism, a characteristic of PCOS (Peitsidis and Agrawal, 2010; Almawi et al., 2016).

Based on the result of Mann-Whitney test, there was a significant difference in VEGF expression in the endometrium between women with PCOS and normal women. The VEGF expression increased in 
the endometrial of PCOS. This result is in line with a study carried out to 80 patients that the level of VEGF of serum was significantly higher in women with PCOS (Tahergorabi et al, 2019).

Several other studies have shown that the concentration of VEGF protein in follicular fluids or follicular fluids (FF) was higher than the serum concentration, indicating that the source of increased serum VEGF was the ovary (Scotti et al., 2014).

VEGF was an endothelial cell mitogen and heparin-binding glycoprotein which played a role as a major factor during physiological and pathological angiogenesis. One of the mechanisms that might explain this condition was PCOS was associated with chronic inflammation. It could cause abnormal angiogenesis in ovarian tissue. In addition, increased level of VEGF in the serum of patients with PCOS could be caused by an increase in the number of granulosa lutein cell that actively secreted and expressed the gene regulated from these cells, because granulosa lutein cells were the most important source of VEGF in the female reproductive system (Harada et al, 2010).

During periods, VEGF expression in the endometrium occurred most frequently in the secretory phase during the implantation (Chenet al., 2015). Increased VEGF would disrupt the adhesion process, thus disrupting the subsequent processes such as invasion, decidualization, and implantation process (Yoshii et al., 2013).

The VEGF concentration of serum would increase during the luteal phase of periods. Increased expression of VEGF in the dense hyperchaotic stroma in PCOS was associated with increased ovarian stroma blood flow in women with PCOS. It caused uncontrolled follicular growth and intense vascularization which could lead to abnormal growth of the theca interna, where there was androgen steroidogenesis. The increase in vascularity in the dense stroma in women with PCOS has been confirmed by several studies that showed that the rate of Doppler blood flow increased in women with PCOS (Peitsidis and Agrawal, 2010).

A significant difference was found between the group of women with PCOS and normal women. The BMI that was obese was higher in the PCOS group. Obesity was associated with insulin resistance and hyperinsulinemia compensation. Insulin played a role as a co-gonadotropin to stimulate ovarian androgen production. Several conditions of hyperinsulinemia that were very resistant to insulin in women have been linked to obvious hyperandrogenemia. A small increase in circulating ovarian androgens was found by infusing insulin in women with normal ovaries, as well as when women with type I diabetes were treated with insulin. Antidiabetic drugs that reduced insulin levels or increase insulin sensitivity has been linked to decreased circulating androgen levels and increased ovulation levels. Some growth factors and other inflammatory factors increased in obesity and could stimulate the production of excess ovarian androgens or inhibited aromatization of androgens to estrogen (Repaci et al., 2011).

In PCOS, obesity could potentiate hyperandrogenemia through several mechanisms. Insulin resistance and/or hyperinsulinemia have been associated with direct hypothalamic effects which might support iregular gonadotropin secretion. Obesity could affect the peripheral metabolism of sex steroids or sex steroid regulators. Besides the effects of insulin resistance mediated by hyperinsulinemia, increased adiposity caused faster cortisol metabolism, thus resulting hypothalamus-hypophysis-adrenal-axis(HHAA) hyperactivation. Therefore, it increased dehydroepiandrosterone (DHEA) synthesis. Competitive binding of glucocorticoid receptors of progesterone derivatives caused a decrease in 
cortisol binding with glucocorticoid receptors, thus strengthening the activation of HHAA. High adiposity also caused greater aromatase expression in adipocytes, thus allowing an increase in extraovarian estrogen synthesis. This caused an increase in the LH/FSH ratio. It also encouraged glucocorticoid hyperplasia and theca cells, so that it synthesized progesterone and testosterone in greater amounts (Legro et al., 2012; Rojas et al, 2014).

Androgen activity was related to peripheral androgen metabolism and binding proteins such as sex hormone-binding globulin (SHBG) which limited the bioavailability of peripheral androgens. Androgen metabolism in peripheral regions affected androgen activity. For example, androgen was considered to have peripheral aromatization in several locations which could have local effects associated with PCOS and also have systemic activity if these metabolites were excreted. Adipose tissue contained aromatase which could increase bioactive estrogen levels from androgens. It was released into the circulation. It could also cause an increase in the level of estrone found in women with PCOS. This increase might be one of the mechanisms that contribute to anovulation conditions. This condition was considered to contribute to the estrogen condition which supported the development of endometrial hyperplasia. The distribution and relative amount of fat could affect the metabolic phenotype and reproduction of women with PCOS. Women with increased central adiposity and visceral fat generally had higher condition of metabolic dysfunction, inflammation, and hyperandrogenism (Legro et al., 2012).

Carbohydrate-rich diets could cause oxidative stress in the circulation of mononuclear blood cells, thus causing chronic inflammation. Lipid-rich diets had been reported to increase testosterone synthesis and decrease SHBG regulation, thus resulting hyperandrogenemia. Both short-term and long-term high-fat diets and low-fiber had been linked to hyperandrogenemia. It possibly worked through hyperinsulinemia mechanisms related to food intake, thus reducing SHBG synthesis and increasing the availability of androgens (Mukherjee and Maitra, 2010). Nevertheless, new mechanisms showed a direct effect of diet on disorders of ovarian physiology. Advanced glycation end products (AGE) are cytotoxic metabolites derived from carbohydrate metabolism that was disrupted, which could also be obtained exogenously from a variety of foods (Diamanti-Kandarakis et al, 2007). AGE deposition in ovarian tissue induced oxidative stress and disruption of structural modification due to molecular crosslinking, thus causing damage to all types of ovarian cells and changed all aspects of its function. Hyperandrogenemia also inhibited the activity of glyoxalase-I, which was an important enzymatic scavenging system for 2-oxoaldehyde, including the main precursor of AGE (Kandaraki et al, 2012).

\section{AUTHOR CONTRIBUTION}

Ricky Bernadi Saputra, Uki Retno Budihastuti, Sri Sulistyowati, Eriana Melinawati, and Supriyadi Hari Respati did study concept, examined vascular endothelial growth factor expression, and drafted the manuscript.

\section{CONFLICT OF INTEREST}

The author stated that this study was conducted without any relationship to financial or commercial factors that could be interpreted as a conflict of interest.

\section{FUNDING AND SPONSORSHIP}

This study used funds from Non-Tax State Revenue (PNBP), Sebelas Maret University. 
Saputra et al. / The Difference of Vascular Endothelial Growth Factor Expression

\section{ACKNOWLEDGEMENT}

The researchers thank all the Obstetrics and Gynecology staff at Dr. RSUD Hospital Moewardi Surakarta who have allowed us to conduct this study, as well as all patients who were willing to be the subject of the study. The researchers were also grateful because the researchers were allowed to share the knowledge and pleased to collaborate during the study.

\section{REFERENCE}

Almawi WY, Gammoh E, Malalla ZH, AlMadhi SA (2016). Analysis of VEGFA Variants and Changes in VEGF Levels Underscores the Contribution of VEGF to Polycystic Ovary Syndrome. PLoS ONE 11(11): eo165636. https://doi.org/10.1371/journal.pone.0165636.

Diamanti-Kandarakis E, Piperi C, Korkolopoulou P, et al. (2007). Accumulation of dietary glycotoxins in the reproductive system of normal female rats. $\mathrm{J}$ Mol Med (Berl). 2007;85(12):1413-1420. doi:10.1007/s00109-007-0246-6.

Harada M, Peegel H, Menon KM (2010). Expression of vascular endothelial growth factor A during ligand-induced downregulation of luteinizing hormone receptor in the ovary. Mol Cell Endocrinol. 328(1-2): 28-33. https://dx.doi.org/10.1016\%2Fj.mce.2010.06.015.

Kandaraki E, Chatzigeorgiou A, Piperi C, Palioura E, Palimeri S, Korkolopoulou $\mathrm{P}$, Koutsilieris M, Papavassiliou AG (2012). Reduced ovarian glyoxalase-I activity by dietary glycotoxins and androgen excess: a causative link to polycystic ovarian syndrome. Mol Med: 18(1): 1183-1189. https://dx.doi.org/10.2119\%2Fmolmed.2012.00293.

Lai TH, Vlahos N, Shih IeM, Zhao Y (2015). Expression Patterns of VEGF and Flk-1 in Human Endometrium during the Menstrual Cycle. J Reprod Infertil.
16(1): 3-9. https://www.ncbi.nlm.nih.gov/pubmed/25717429.

Legro RS (2012). Obesity and PCOS: Implications for diagnosis and treatment. Semin Reprod Med, 30(6): 496-506. doi: 10.1055/s-0032-1328878.

Mukherjee S, Maitra A (2010). Molecular \& genetic factors contributing to insulin resistance in polycystic ovary syndrome. Indian J Med Res. 131:743-760.

Peitsidis P, Agrawal R (2010). Role of vascular endothelial growth factor in women with PCO and PCOS: A systematic review. Reproductive BioMedicine Online (20): 444-452. https://doi.org/10.1016/j.rbmo.2010.01.007.

Repaci A, Gambineri A, Pasquali R (2011). The role of low-grade inflammation in the polycystic ovary syndrome. Mol Cell Endocrinol, 335(1): 30-41. https://doi.org/10.1016/j.mce.2010.08.002.

Ring M (2017). Polycystic ovarian syndrome, menopause, and osteoporosis. Prim Care Clin Office Pract. (44): 377-398. https://doi.org/10.1016/j.pop.2017.02.012.

Rojas J, Chávez M, Olivar L, Rojas M, Morillo J, Mejías J, Calvo M, Bermúdez V (2014). Polycystic ovary syndrome, insulin resistance, and obesity: navigating the pathophysiologic labyrinth. Int $\mathrm{J}$ Reprod Med. 719050. doi:10.1155/2014/719050.

Sachdeva G, Gainder S, Suri V, Sachdeva N, Chopra $S$ (2019). Obese and non-obese polycystic ovarian syndrome: comparison of clinical, metabolic, hormonal parameters, and their differential response to clomiphene. Indian J Endocrinol Metab, 23(2): 257-262. doi:10.4103/ijem.IJEM_637_18.

Schneider D, Gonzalez JR, Yamamoto M, Yang J, Lo JC (2019). The Association of Polycystic Ovary Syndrome and Gestational Hypertensive Disorders in a 
Saputra et al. / The Difference of Vascular Endothelial Growth Factor Expression

Diverse Community-Based Cohort. J Pregnancy, 9847057: 1-6. https://dx.doi.org/10.1155\%2F2019\%2F9847057

Scotti L, Parborell F, Irusta G, De Zuñiga I, Bisioli C, Pettorossi H, Tesone M, Abramovich D (2014). Platelet-derived growth factor BB and DD and angiopoietin1 are altered in follicular fluid from polycystic ovary syndrome patients. Mol Reprod Dev. 81(8): 748-756. https://doi.org/10.1002/mrd.22343.

Sumapraja K, Pangastuti N (2011). Profile of Policystic Ovarian Syndrome Patients in Dr. CiptoMangunkusumo General Hospital Jakarta March 2009 -March 2010. Indonesian Journal of Obstetrics and Gynecology, 35(1).

Tahergorabi Z, Salmani F, Jonaidabad SH, et al. (2019). Association of serum levels of vascular endothelial growth factor and thrombospondin-1 to body mass index in polycystic ovary syndrome: a case-control study. Obstet Gynecol Sci. 62(6): 420-428. doi:10.5468/ogs.2019.62.6.420.

Yoshii N, Hamatani T, Inagaki N, Hosaka T, Inoue O, Yamada M, Machiya R, Yoshimura Y, Odawara Y (2013). Successful implantation after reducing matrixmetalloproteinase activity in the uterine cavity. Reprod Biol Endocrinol, 11:37. https://dx.doi.org/10.1186\%2F14777827-11-37.

Zhao DM, Shan YH, Li FH, Jiang L, Qu QL (2019). Correlation between endometrial receptivity with expressions of IL-1 and VEGF in rats with polycystic ovary syndrome. Eur Rev Med Pharmacol Sci, 23(13): 5575-5580. doi:10.26355/eurrev_201907_18291. 